This item was submitted to Loughborough's Research Repository by the author.

Items in Figshare are protected by copyright, with all rights reserved, unless otherwise indicated.

In vivo determination of the effect of shoulder pads on tackling forces in rugby

PLEASE CITE THE PUBLISHED VERSION

http://dx.doi.org/10.1080/02640410801910319

PUBLISHER

Routledge (@ Taylor \& Francis)

VERSION

AM (Accepted Manuscript)

LICENCE

CC BY-NC-ND 4.0

REPOSITORY RECORD

Pain, Matthew T.G., Felix Tsui, and Stephen Cove. 2019. "In Vivo Determination of the Effect of Shoulder Pads on Tackling Forces in Rugby”. figshare. https://hdl.handle.net/2134/6543. 
This item was submitted to Loughborough's Institutional Repository (https://dspace.lboro.ac.uk/) by the author and is made available under the following Creative Commons Licence conditions.

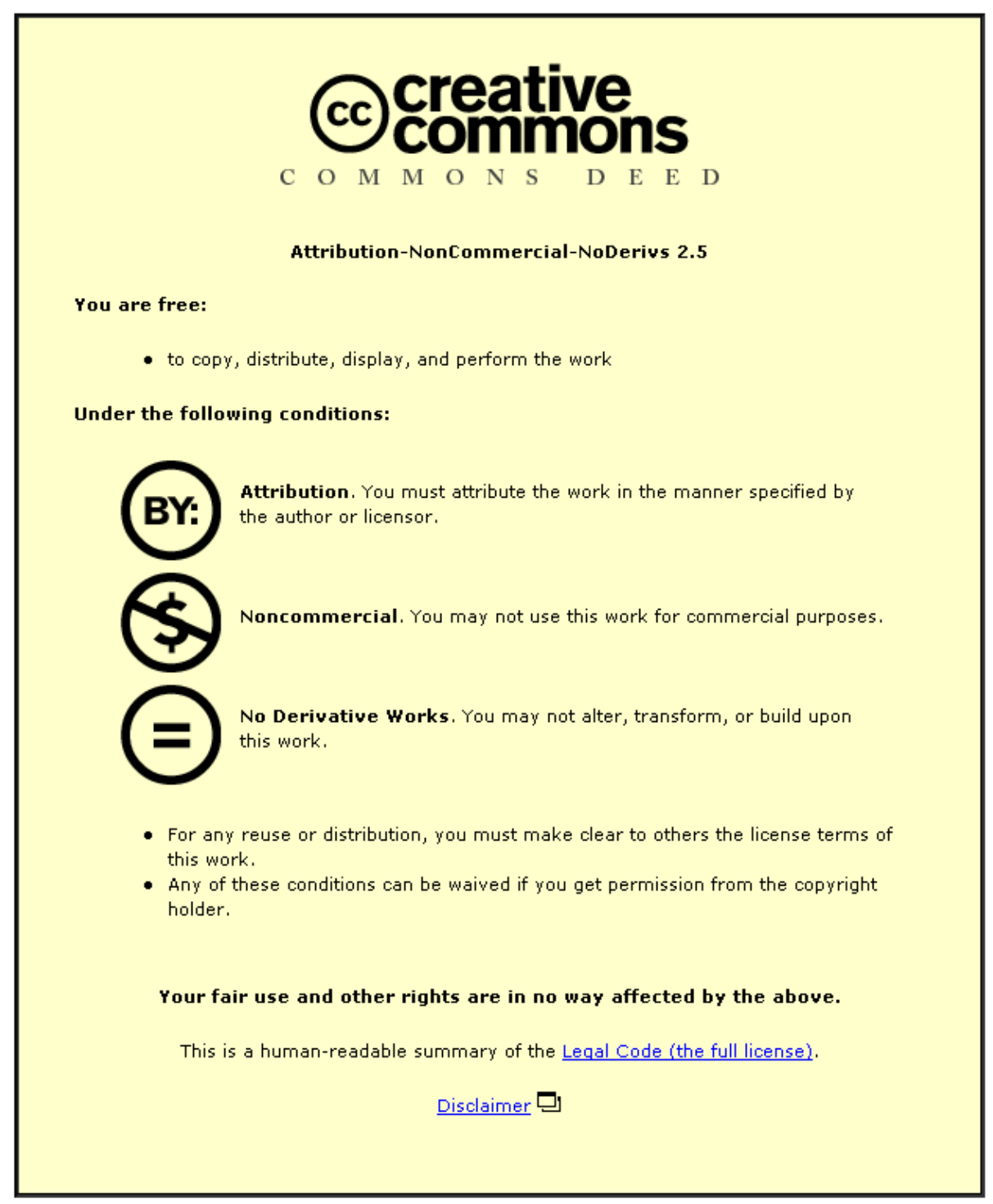

For the full text of this licence, please go to: http://creativecommons.org/licenses/by-nc-nd/2.5/ 


\title{
In vivo determination of the effect of shoulder pads on tackling forces in rugby
}

\author{
Mathew G. Pain, Felix Tsui, and Stephen Cove \\ School of Sport and Exercise Sciences, Loughborough University,UK
}

\begin{abstract}
Tackling in rugby is now a major cause of injury. The use of rugby shoulder pads is intended to reduce injury from front-on tackles, though the pad's ability to reduce injury has not been examined. This paper strives to present a novel method, using Tekscan sensors, for measuring in vivo impact intensities during an actual front-on tackle in order to assess the effectiveness of rugby shoulder padding in reducing peak force during impact. It was hypothesised that padding would not significantly reduce peak impact force. Rugby pads were instrumented with thin film force sensors to measure impact intensities during tackles with and without pads. Sensors were statically calibrated then dynamically calibrated using force plate data. Results showed that the pad significantly reduced peak impact force by up to $35 \%$ when impacted with an object and by $40 \%$ overall for all tackles. The hypothesis that the shoulder pad could not significantly reduce peak force at impact was rejected since the pad reduced peak force by $41 \%$ in tackles with a run-up and $40 \%$ overall for all tackles. However, this reduction in force was localised directly above the acromioclavicular joint, while forces in the surrounding areas were not reduced.
\end{abstract}

Keywords: rugby, tackle, pads, impact

\section{Introduction}

Rugby is a fast-paced, full-contact sport which does not require the use of protective equipment. Injury incidence in rugby is much higher than that reported in professional ice hockey, soccer and cricket (Brooks, Fuller, Kemp \& Reddin, 2005a; Brooks, Fuller, Kemp \& Reddin, 2005b ). Recent research in the field of rugby union has highlighted a shift from scrummage-related injuries to tackle-induced injury (Wilson, Edwards, Warren \& Scarangella, 1999; McIntosh, 2005). In fact, the tackle has been identified as the major cause of increased incidence of spinal injury (Quarrie, Cantu \& Chalmers, 2002) and overall injury increases in rugby union (Garraway, Lee, Hutton, Russell \& Macleod, 2000). In general, 50\% of all injuries are caused from either making or receiving a tackle (Taylor \& Coolican, 1987; Lee \& Garraway, 1996; Garraway \& Macleod, 1995; McIntosh, 2005). Injuries sustained during front-on tackles are mainly to the shoulder, particularly at the acromioclavicular joint (Wickiewicz, Edwards, Warren \&Scarangella, 1993; Wilson et al., 1999), with shoulder dislocations/instabilities accounting for the second highest number of missed days in rugby union (Brooks et al., 2005a,b).

Research into protective equipment in rugby has mainly focused on the influence of headgear, shin guards and mouth guards in reducing injury (Wilson, 1998; McIntosh, McCrory \& Finch, 2004; Marshall, Loomis, Waller, Chalmers, Bird, Quarrie et al., 2004). The introduction of shoulder pads was meant to decrease the incidence of shoulder injury during front-on rugby tackles, but its performance has not been quantitatively assessed. International Rugby Board (IRB) regulations do not outline the level of protection required of shoulder pads, but stipulates three conditions to be met before the pads can be used in matches. The padding material cannot exceed $45 \mathrm{~kg} \cdot \mathrm{m}^{-3}$ in density and must provide maximum coverage to the stenoclavicular, acromioclavicular (AC) and glenohumeral joints. Pads must also be subjected to an 
IRB-prescribed hammer and anvil test, which involves dropping a rigid, flat striking surface ( $5 \pm 0.02 \mathrm{~kg}$ ) onto a pad resting on a steel anvil. However, this test only provides information on the material properties of the pad itself and not the amount of protection provided to the user.

In a tackle, the padding is not sandwiched between two rigid metal bodies but between two sets of non-linear, viscoelastic bodies. The net effect of the padding is dependent on the resultant viscoelastic properties of the whole system and the total energy imparted during impact. Ideally, for padding to be effective and offer maximum protection, it needs to be tuned to the specific task it aims to defend against; that is, the padding needs to be tested under realistic loading regimes. Milburn, Wilson \& Chalmers (2001) used a medicine ball, a much less stiff impactor than that used in hammer and anvil tests, which was claimed to better represent soft tissue. It was found that IRB-approved shoulder pads did not provide a significant improvement in peak force reduction. As expected, the choice of impactor had a direct influence on the impact-mitigating performance of the shoulder pad. This suggested that more biofidelic impactors should be used in further tests, though the most meaningful results would likely be obtained during an actual tackle.

The lack of detailed understanding into the mechanics of shoulder pads is further highlighted by Gerrard (1998) which discussed, on two occasions, that the shoulder pads acted to disperse applied forces. During impact, the foam materials deform in a point-elastic manner and provide minimal dispersion of the load. The reduction in force is mainly due to the increased time of contact during the impact as the pad deforms causing lower decelerations. As such, the benefit of shoulder pads as protectors against superficial soft tissue trauma, as opposed to their ability to prevent more serious damage, has been heavily debated (Gerrard, 1998; McIntosh, 2005). The general consensus was that serious injury prevention from the padding is unlikely but this was not conclusive. This can be problematic as players may be under the impression that they are afforded significantly more protection, which can lead to an increase in the intensity of tackles.

Peak impact force from scrummages have been measured, ranging from 2800 4000 N (Cohen \& Stiff, 1979; Milburn, 1990; Quarrie \& Wilson, 200), but directly measuring the impact intensity of a tackle was thought to be an impossible task (Milburn, 1995). With increasing clinical applications, thin-film, flexible force sensors have become more readily available. Tekscan ${ }^{\circledR}$ sensors feature piezoresistive technology, consisting of rows and columns of semi-conductive paint which intersect at points called 'sensels'. When force is applied to the sensor, a change in voltage is measured by each individual sensel which is proportional to the applied force. This system potentially allows for in vivo measurements to be taken during player-on-player tackles. However, this methodology is still not without its own limitations of temporal and spatial resolution and sensitivity which requires the development of a method for dynamic calibration and separate tests on the shoulder pad involving different stiffness impactors on a force plate.

Of course whether the pads are tested in a factory or in vivo the results still only apply to the force attenuation properties of the pads under different impact conditions, not what effect they could have on actually preventing injuries during matches and training. Injuries are caused from range of mechanisms such as: blunt trauma, shearing, twisting actions through excessive ranges of motion, high pressures, high loading rates, and high rates of pressure change, and are also dependent on body geometry, preparedness and musculature stiffness. The multi-factorial nature of injury 
potential means that the effectiveness of shoulder pads in reducing actual injuries can only be properly determined by controlled longitudinal epidemiological studies. However, the manufacturers and IRB introduced them with the goal of making things safer, and given there construction, they are only likely to have a marked affect on force, rate of force rise and reducing surface shear. As the sensors cannot measure shear forces this not only precludes analysing surface shears but will also lead to an underestimation of the total forces present during tackles.

This paper strives to present a novel method, using Tekscan sensors, for measuring in vivo impact intensities during an actual front-on tackle in order to assess the effectiveness of rugby shoulder padding in reducing peak force during impact. The assumption as to whether any reduction in force will lead to lower injury rates will not be explicitly addressed. It is hypothesised that the rugby shoulder padding will be ineffective in reducing peak force during the tackle.

\section{Methods}

Calibration

Static calibration of the sensors followed the procedure outlined by Tekscan. A controlled force was applied evenly across the surface of the Tekscan F-Socket 9811 force sensor (Boston, MA), sampling at $250 \mathrm{~Hz}$, through a custom-designed airtight calibration bladder attached to a pressure generator. The F-Socket 9811 sensor has a 6 x 16 transducer array of sensels, forming an effective measurement area of $203 \mathrm{~mm} \mathrm{x}$ $76 \mathrm{~mm}$. The generator was programmed to apply $655 \mathrm{KPa}$ (95 psi) and the software performed a linear point-to-point calibration from no-load, correlating to a sensor saturation force of $10176 \mathrm{~N}$ (106 N per sensel).

Due to the low sampling rate of the Tekscan force sensor and its method of multiplexing data from the columns of sensels, a dynamic calibration was necessary. Impacts were generated by dropping three objects of varying stiffness onto the Tekscan sensor, centred on top of a Kistler 2981B12 force plate, sampling at $2000 \mathrm{~Hz}$. This allowed for simultaneous measurements of force-time histories during impact as recorded by Tekscan and the force plate. The objects used were a $7.26 \mathrm{~kg}$ indoor shot, $10 \mathrm{~kg}$ medicine ball and $5 \mathrm{~kg}$ weight belt (Figure 1). Drop heights for each object increased in increments of $10 \mathrm{~cm}$ from $20 \mathrm{~cm}$ to $70 \mathrm{~cm}$ from a custom drop rig.

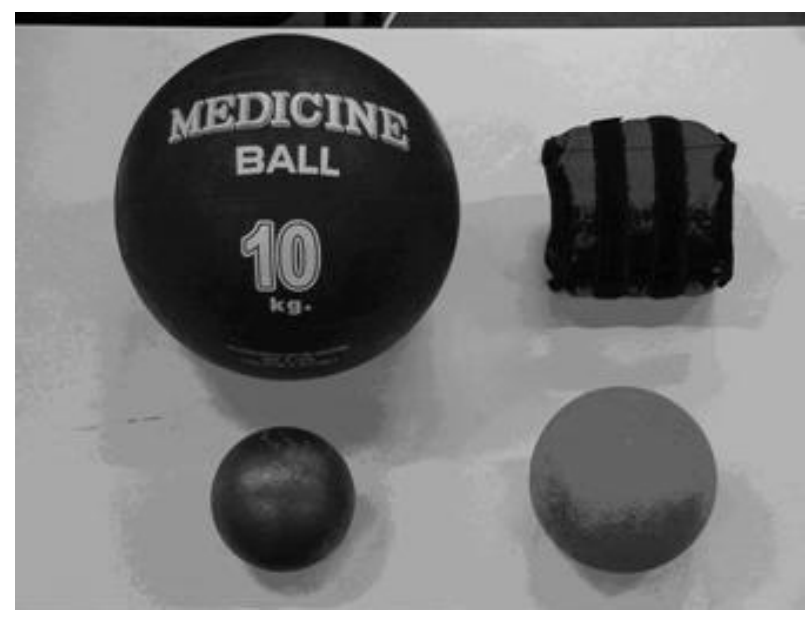

Figure 1. Objects used to assess the impact attenuation of the rugby pad. From top left, clockwise: medicine ball, weight belt, indoor shot and outdoor shot. 
Force plate data were linearly regressed against the Tekscan data. Each regression line was fitted through the origin and the gradient of the regression line was used as the calibration coefficient for the Tekscan data. The object with a Force - Time curve that had the most similar time to peak force as the tackles was used to scale the tackle data. Time to peak force was measured from time-zero, set one frame before the noise threshold ( $5 \mathrm{~N}$ ) was exceeded, to the frame in which the peak force was captured.

\section{Impact Attenuation Properties of Shoulder Pad}

A shoulder pad ( $1 \mathrm{~cm}$ thick, $30 \mathrm{~kg} \cdot \mathrm{m}^{-3}$ density) from a KooGa ${ }^{\circledR}$ Warrior Upper Body Protective Vest (Figure 2) was removed and subjected to isolated impact testing. Impacts were generated using the same methods as the dynamic calibration with the same objects plus an outdoor shot. The force plate, set at $2000 \mathrm{~Hz}$, recorded force from objects dropped onto the pad and directly onto the plate. Drop heights for the padded condition were adjusted to account for the pad thickness, to ensure the same impact velocities at contact. The outdoor shot was not dropped above $60 \mathrm{~cm}$ to avoid overload and damaging the force plate. For each object, the percentage decrease in peak impact force from no-pad to pad was calculated for each drop height. An 8-point average was also taken around each peak impact force for both the pad and no-pad conditions. This helped examine the effects of sampling at $250 \mathrm{~Hz}$ on peak force for the different impactors. As the Tekscan multiplexes over the whole 4 millisecond sample time, this method was used rather than re-sampling the force plate data at $250 \mathrm{~Hz}$ since the force plate takes a single reading per sample time.
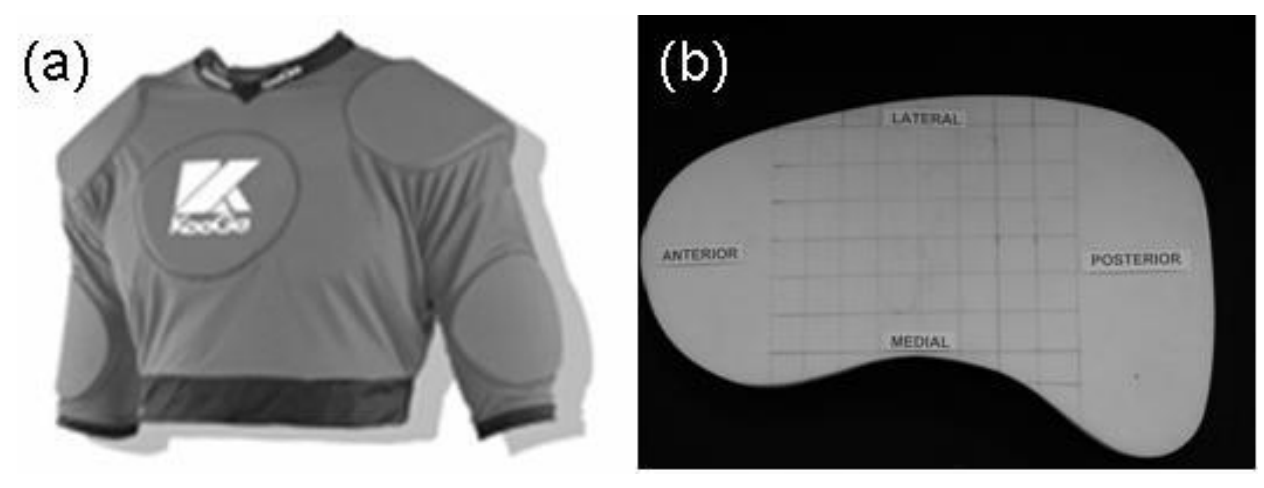

Figure 2. (a) KooGa Warrior padded pest; (b) Shoulder pad.

\section{Tackles}

Six male rugby players $(m=83.0 \pm 13.0 \mathrm{~kg})$ provided informed consent in accordance with the protocol outlined by the Loughborough University Ethical Advisory Committee prior to testing. After a self-guided warm-up, that included practice tackles, each participant performed two sets of three front-on tackles against a stationary opponent. Each set consisted of one low-velocity tackle, performed from a crouch, and two higher-velocity tackles, delivered after a four to five pace run-up. For consistency a tackle zone was marked out with tape on the right thigh of the tackled participant (Figure 4) and instructions were given from a qualified coach to perform a classic front on tackle. Protective shoulder padding was worn by the tackler in one set of tackles and the order of the sets was randomised. The KooGa vest was customised to safely hold the Tekscan system in place (Figure 3). During tackles without padding, the sensor was placed in the customised vest on a paper template to keep the sensor in 
place. All shoulder forces during tackles were recorded at $250 \mathrm{~Hz}$. Pre-load from wearing the pad and from sensor curvature was subtracted from the measured raw force. The location of the sensor relative to the shoulder was examined in the static condition and while the forces due to curvature were recorded. All tackles were recorded in two dimensions at $500 \mathrm{~Hz}$ within a calibrated plane using a Phantom digital camera positioned perpendicular to the plane (Figure 4). The tackler's hip and shoulder velocities were calculated at impact from this camera. A second phantom camera recording at $500 \mathrm{~Hz}$ with an oblique view down onto the shoulder pad was used to qualitatively determine pad motion during impact (Figure 4).

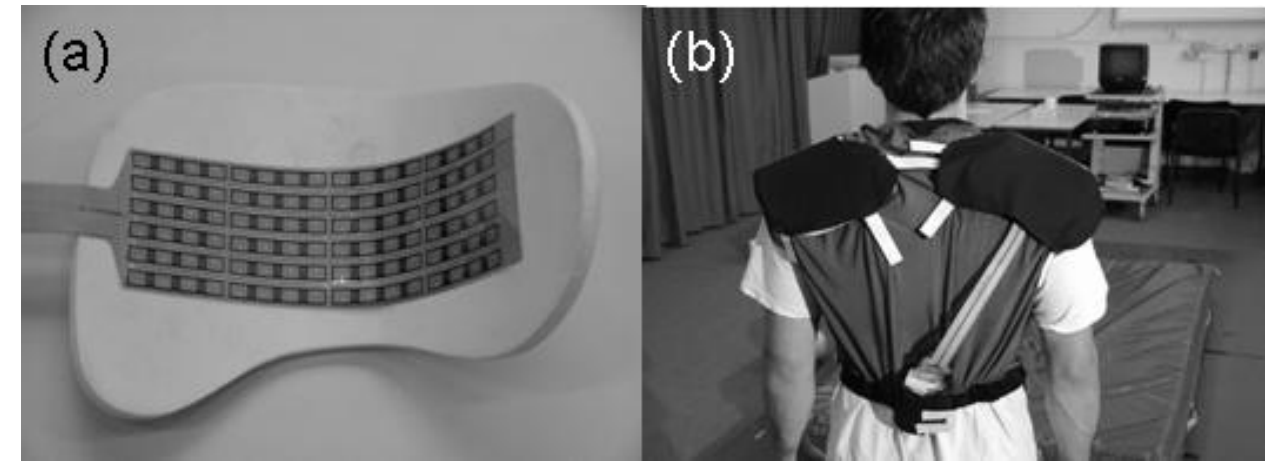

Figure 3. (a) Padding from Kooga ${ }^{\circledR}$ shoulder pad instrumented with F-Socket 9811 sensor; (b) Customised vest with Tekscan.

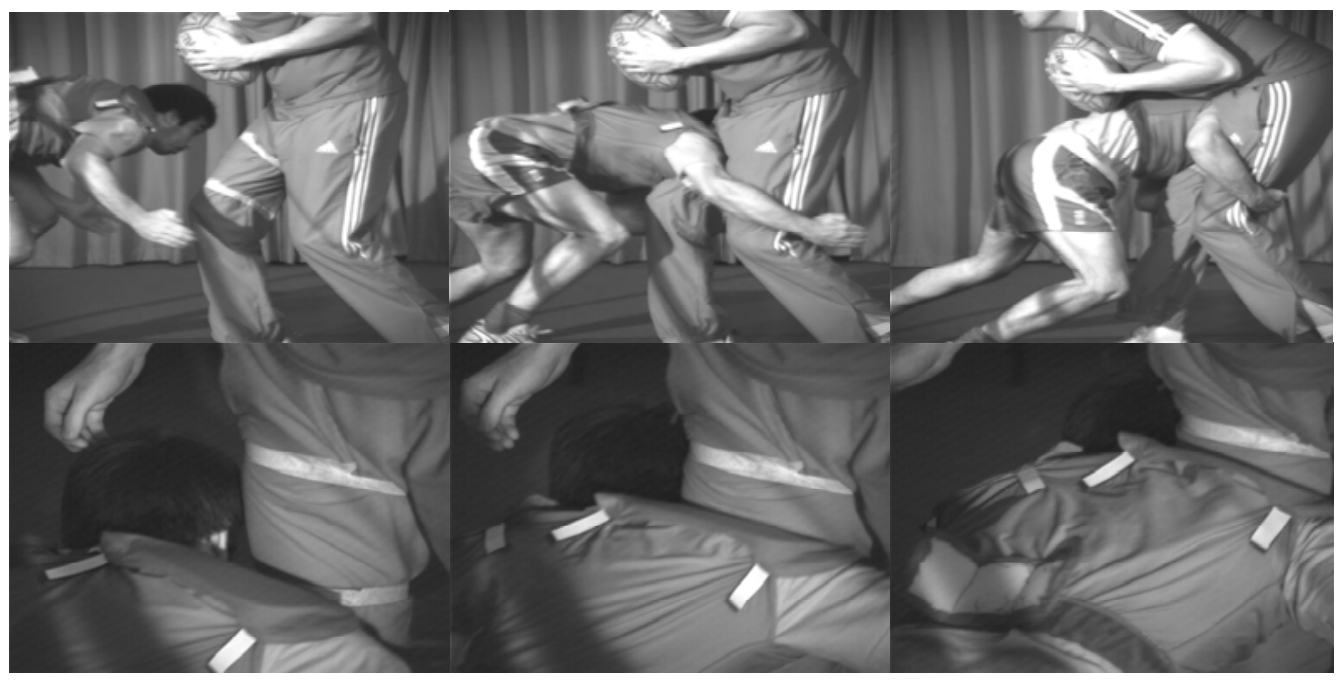

Figure 4. Selected still images from the Phantom hi speed cameras used to calculate impact velocity and qualify pad movement.

\section{Statistics}

Sample means of peak impact force in isolated pad testing and rugby tackles were compared in conditions with and without padding using a paired sample T-test with alpha set at 0.05 . 


\section{Results}

\section{Calibration}

The raw data for each set of impacts along with the regression equation and $\mathrm{R}^{2}$ values are plotted in Figure 5. The calibration coefficient for the indoor shot, medicine ball and weight belt was 3.68, 2.36 and 2.17, while the average time to peak force for each object was $0.007,0.010$ and $0.008 \mathrm{~s}$, respectively.
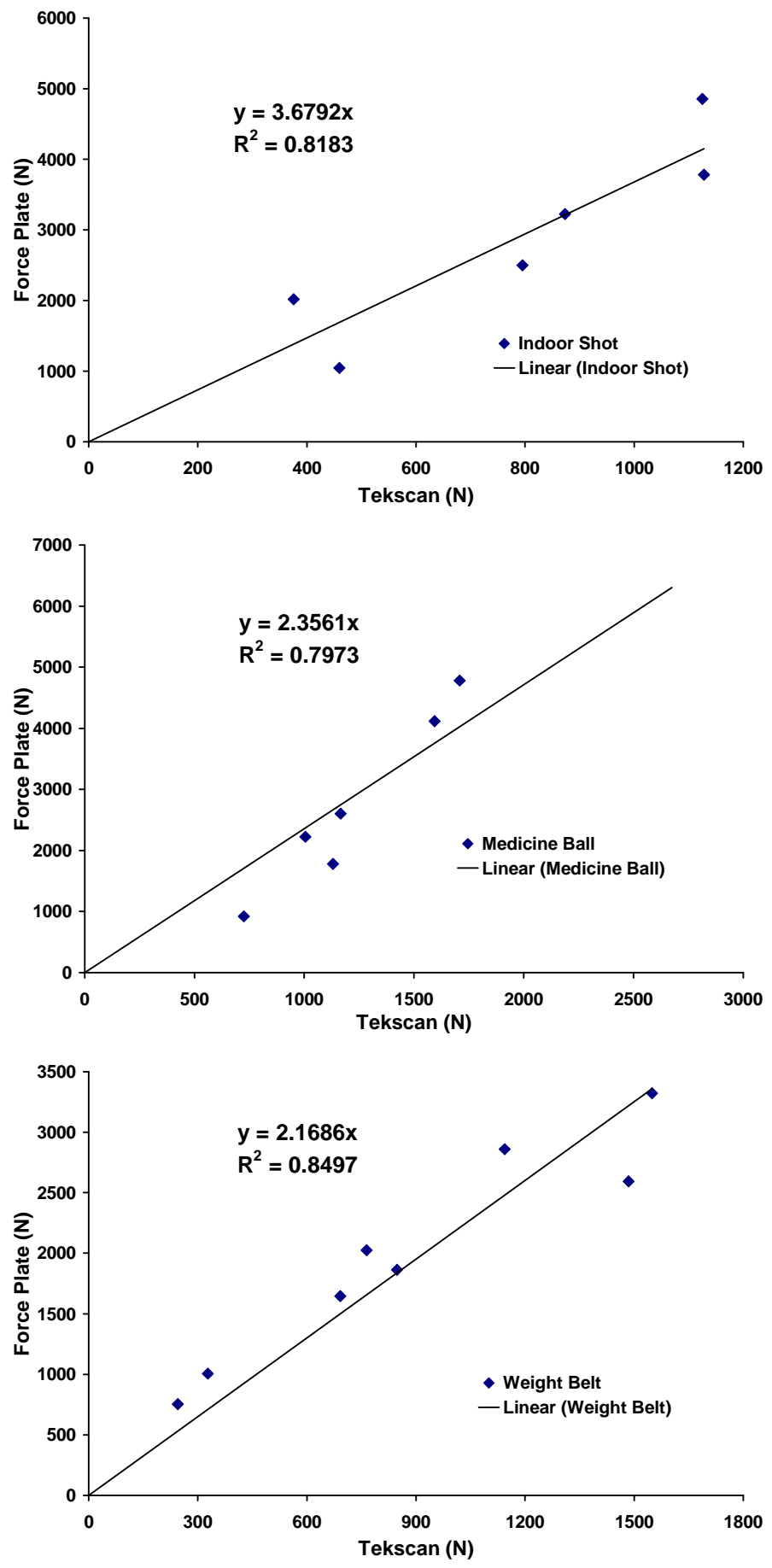

Figure 5. Dynamic calibration curves for the indoor shot, medicine ball and weight belt with regression line equation and $\mathrm{R}^{2}$ value. 


\section{Impact Attenuation}

The peak forces with padding ranged from $1030 \mathrm{~N}$ to $10300 \mathrm{~N}$ and without padding ranged from $1110 \mathrm{~N}$ to $15900 \mathrm{~N}$. Table 1 summarises the percentage decrease in peak force from testing the pad on the force plate at $2000 \mathrm{~Hz}$ and $250 \mathrm{~Hz}$. At 2000 $\mathrm{Hz}$, the shoulder pad significantly decreased the average peak impact force from the outdoor shot put and medicine ball by 35.4\% ( $\quad<<0.05)$ and 3.1\% (p = 0.02), respectively. However, at $250 \mathrm{~Hz}$, only the peak impact force from the outdoor shot put was significantly reduced from the no-pad to pad condition. Adjusting the sampling frequency from $2000 \mathrm{~Hz}$ to $250 \mathrm{~Hz}$ lowered the reduction in peak force with and without the pad for the outdoor shot from $35 \%$ to $12 \%$. Other impactors' peak forces were altered by only a few percent and this highlights the sensitivity of the sampling rate during impact.

Table 1. Average percentage decrease in peak impact force with the shoulder pad placed on the force plate.

\begin{tabular}{lcc}
\hline & \multicolumn{2}{c}{ Decrease in Peak Force (\%) } \\
\hline & $2000 \mathrm{~Hz}$ & $250 \mathrm{~Hz}$ \\
Outdoor Shot Put & 35.4 & 12.4 \\
Indoor Shot Put & 1.5 & 1.2 \\
Medicine Ball & 3.1 & 1.8 \\
\hline Weight Belt & 2.5 & -1.9 \\
\hline
\end{tabular}

\section{Tackles}

With regard to the impact velocities of the shoulder and hip there were no major changes with and without shoulder pads for any individual. Shoulder velocities without pads were $4.5 \pm 0.8 \mathrm{~ms}^{-1}$ and $3.2 \pm 0.5 \mathrm{~ms}^{-1}$ for the run and crouch respectively and with pads they were $4.4 \pm 0.7 \mathrm{~ms}^{-1}$ and $3.5 \pm 0.5 \mathrm{~ms}^{-1}$. Hip velocities without pads were $4.6 \pm 1.0 \mathrm{~ms}^{-1}$ and $2.4 \pm 0.5 \mathrm{~ms}^{-1}$ for the run and crouch respectively and with pads they were $4.4 \pm 1.0 \mathrm{~ms}^{-1}$ and $2.8 \pm 0.3 \mathrm{~ms}^{-1}$. There were no significant differences between either hip or shoulder velocities in the two conditions for either the crouch or run up $(0.1<\mathrm{p}<0.9)$.

Average time to peak force for all tackles was $0.082 \mathrm{~s}$ (range $0.036-0.172 \mathrm{~s}$ ), and a significant difference was not found between tackles made with and without pads. Of the three calibration objects, the time characteristics of the medicine ball best represented the tackle and its calibration coefficient was used to scale the raw data. A summary of the adjusted peak forces appear in Table 2. Force-Time profiles of two representative tackles - one with the pad and one without - are shown in Figure 6. 
Table 2. Adjusted peak impact forces, scaled using the calibration coefficient of the medicine ball, expressed in Newtons and Body Weights. The first entry for each participant represents a tackle from the crouched position and the latter two from a run-up.

\begin{tabular}{ccccc}
\hline Participant & \multicolumn{2}{c}{ Peak Force (N) } & \multicolumn{2}{c}{ Peak Force (Bodyweights) } \\
& With Pad & Without Pad & With Pad & Without Pad \\
1 & 634 & 773 & 0.86 & 1.04 \\
& 777 & 1027 & 1.05 & 1.39 \\
2 & 665 & 1155 & 0.90 & 1.56 \\
& 755 & 1095 & 1.03 & 1.50 \\
& 1016 & 1376 & 1.39 & 1.88 \\
3 & 821 & 1440 & 1.12 & 1.96 \\
& 686 & 1360 & 0.66 & 1.31 \\
& 1422 & 2789 & 1.37 & 2.69 \\
4 & 1679 & 2129 & 1.63 & 2.05 \\
& 398 & 635 & 0.52 & 0.85 \\
& 686 & 835 & 0.91 & 1.11 \\
5 & 724 & 720 & 0.97 & 0.95 \\
& 707 & 472 & 0.78 & 0.52 \\
& 671 & 1195 & 0.75 & 1.33 \\
& 1178 & 1418 & 1.30 & 1.58 \\
& 405 & 581 & 0.56 & 0.80 \\
& 539 & 727 & 0.75 & 1.01 \\
& 596 & 585 & 0.83 & 0.81 \\
\hline \multirow{3}{*}{5} & & & &
\end{tabular}

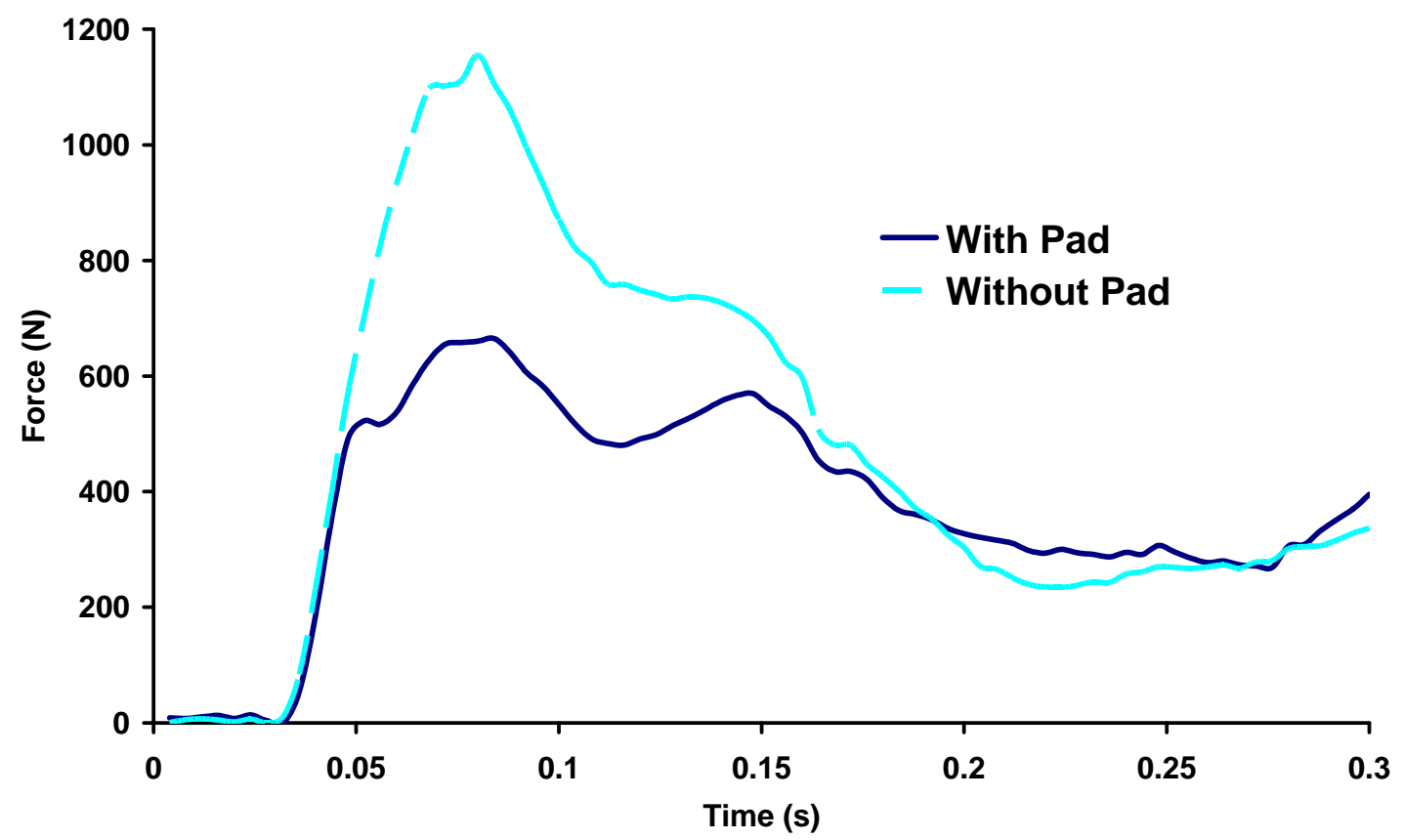

Figure 6. Representative Force-Time profiles for tackles with and without pads.

In running tackles, shoulder pads significantly reduced the peak impact force from $1.53 \pm 0.55 \mathrm{BW}$ to $1.08 \pm 0.28 \mathrm{BW}(\mathrm{p}=0.0017)$. This correlated to a $41 \%$ decrease in peak force when pads were used. Overall, the decrease in peak force was $40 \%$ across all tackles, though a significant difference was not found in crouched tackles ( $\mathrm{p}=$ 0.086). In tackles where no padding was used, the force-distribution curves showed a 
distinct force peak (Figure 7a-c) situated over the area of the acromioclavicular joint. When padding was used this peak was significantly reduced, while force levels around this peak did not exhibit an appreciable change (Figure 7d-f).

(a)

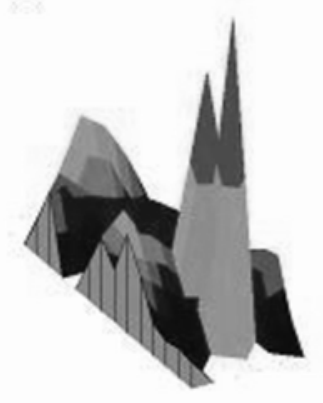

(d)

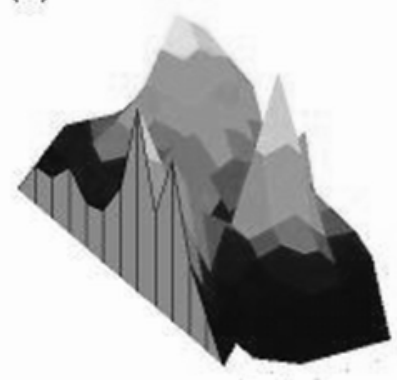

(b)

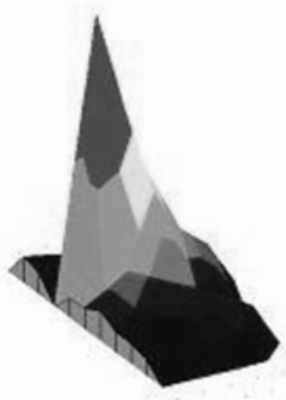

(e)

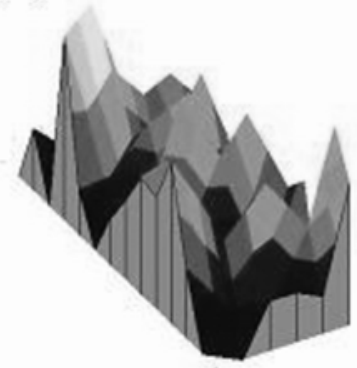

(c)

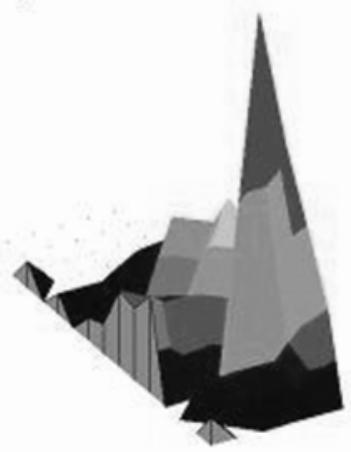

(f)

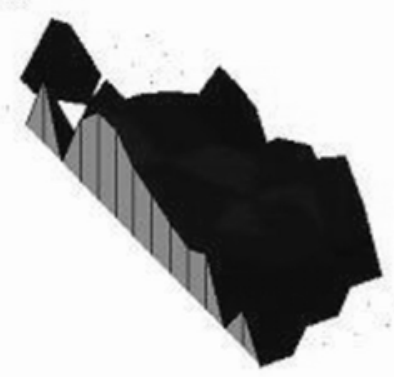

Figure 7. Distribution of impact force during tackle. Conditions (a) - (c) tackles without padding for running tackles from 3 participants.Conditions (d) - (f) display tackles with padding for running tackles from 3 participants.

\section{Discussion}

The aim of this study was to examine the ability of a rugby shoulder pad to attenuate the peak impact force during a front-on rugby tackle. The hypothesis that the shoulder pad could not significantly reduce peak force at impact was rejected since the pad reduced peak force by $41 \%$ in tackles with a run-up and $40 \%$ overall for all tackles.

The isolated pad tests illustrate that the impact response of the pad was sensitive to the particular impact condition - the pad was only effective at mitigating impact from stiffer impactors. Peak impact forces caused by dropping an outdoor shot onto the pad were significantly reduced by an average of 35\%. This test was most similar to the protocol outlined by the IRB for testing shoulder pads. While a rigid impactor and anvil allow for consistent impacts and repeatable results, these apparatus do not have the same mechanical properties as humans. The medicine ball results found a similar percentage decrease to Milburn et al. (2001). However, their 4.5\% reduction in peak force was not significant whereas the $3.1 \%$ reduction found here was, despite being 10 times smaller than the reduction observed for the outdoor shot. The much smaller reductions in peak force for the three less rigid impactors are far less likely to have a mechanical or clinical significance than the 35\% reduction for the outdoor shot.

The time to peak force in tackles without pads was $0.07 \pm 0.04 \mathrm{~s}$ and the time to peak force for the indoor shot, medicine ball and weight belt were $0.007,0.01$ and $0.008 \mathrm{~s}$, respectively. While the medicine ball had the closest time characteristics, it 
still did not accurately simulate the force development rate during a tackle. The force plate was present in all the isolated tests and this immovable steel plate causes a quicker deceleration of the object, leading to a shorter time to peak force, when compared to human tissue. Thus, it is imperative that test rigs feature more human-like material properties when performing biomechanical tests to determine human responses.

Examining at the force distribution curves from actual rugby tackles, rather than just the peak force, gives similar results to the isolated pad tests. Figure 6 shows that padding significantly reduced the peak force spike. However, the levels of force around this spike were not noticeably reduced. Therefore, the isolated pad testing and in vivo tackle measurement suggested two things. First, using a medicine ball may be an appropriate impactor for the majority of the shoulder tackling area, but a far stiffer impactor, such as the outdoor shot, may be necessary to represent the response of the bony impacts. Second, uniform-density padding will not provide the same level of protection to different body parts; that is, different degrees of padding are required over soft tissue and bone. This outlines the importance of in vivo testing, or at the very least, selecting impact objects that simulate human response when attempting to obtain meaningful results about impact attenuation properties of padding.

The force distributions in Figure 7 show that the peak impact force during tackles was reduced by the use of padding, with the peak likely to have been caused by impact of the AC joint. However, there is no evidence to suggest that this actually reduces the severity of injury to this region, particularly in the prevention of dislocations. Dislocations are commonly caused from force transmitting through an abducted arm combined with a rigorous rotational force, which forcibly stretches the supporting muscles and ligaments (Gerrard, 1998). While it is unknown how each force directly contributes to injury, it is unlikely that the pads are effective in minimising rotational force, suggesting that the risk for dislocations may not actually decrease with padding. It is not known if high loads over the AC joint lead directly to injury, but it can cause acute pain. Reducing the load only over the bony parts may reduce the perception of discomfort, but it does not lessen the risk of injury as the majority of the load on the rest of the shoulder is not mitigated. This could contribute to the players making harder tackles as they may be under the impression that the padding will protect against injury.

There are still a number of methodological issues that need improving to examine the effect of shoulder pads in vivo in greater detail. In tackles where no padding was used, the average peak force was $1.35 \pm 0.54 \mathrm{BW}$ (range $0.52-2.69 \mathrm{BW}$ ). This seems rather low as in some cases players were visibly winded and slow to rise after being tackled. These potentially inaccurate force measurements exposed three issues with the Tekscan sensor. Firstly, the sensor area was too small as large forces were generated up to and beyond the sensor boundary (Figure 7). Secondly, despite performing a dynamic calibration, the sensor's method of multiplexing data within a long sampling window (4 ms) may have caused the total impact peak force to be missed. Thirdly, the sensor has a low dynamic response time - a trend found in the F-scan, a similar Tekscan sensor based on the same technology as the F-socket (Sumiya, Suzuki, Kasahara \& Ogata, 1998). These issues can be alleviated with the use of a larger sensor that employs a higher sampling frequency. A further limitation is the inability to measure shear force and the fact that the sensors will produce a response if creased or curved too acutely. When these sensors are used in footwear over similar loading 
regimes the error due to these processes is usually in the order of a few 10's of Newtons at the most and should not have had a major affect on these results.

The density of the KooGa ${ }^{\circledR}$ Warrior Upper Body Protective Pad $\left(30 \mathrm{~kg} \cdot \mathrm{m}^{-3}\right)$ was well within the IRB guidelines $45 \mathrm{~kg} \cdot \mathrm{m}^{-3}$. It could be argued that more pads should have been tested. As all pads must pass the hammer and anvil test, and Milburn (2001) showed pads were not significantly different under softer impact conditions, the results of this study are likely to be applicable to other approved pads. Furthermore, this study concentrated more on providing more realistic impactor conditions than prescribed by the IRB and presenting a novel method of measuring impact forces during actual fronton tackles. The ideas and methodologies expressed here can be used in future testing of a wide variety of rugby shoulder pads.

\section{Conclusion}

It is clear that padding can play an important role in significantly reducing the peak force during a front-on rugby tackle by $41 \%$, although it appears to be limited in its efficacy to the area above the acromioclavicular joint in this study. However, there is no evidence to suggest that this leads to a reduction in injury when playing and training; this can only be properly determined by controlled longitudinal epidemiological studies. For a given pad density, the amount of peak force reduction appears to be related to the stiffness of the impactor. If mechanical testing is to have higher biofidelity, researchers must vary the levels of stiffness in the impactor to model the soft tissue and bone for the part of the body that is used in tackling. Ultimately, the most meaningful results will only be obtained from measuring impact intensities during an actual front-on rugby tackle, similar to the procedure presented in this paper. In vivo measures of tackling force were obtained and changes in force with and without padding were determined but were subject to some measurement limitations. In future in vivo testing should be conducted with a force sensor with a larger measuring area and higher sampling frequency.

\section{References}

Brooks, J.H.M., Fuller, C.W., Kemp, S.P.T. \& Reddin, D.B. (2005a). Epidemiology of injuries in English professional rugby union: part 1 match injuries. British Journal of Sports Medicine, 39, 757-766.

Brooks, J.H.M., Fuller, C.W., Kemp, S.P.T. \& Reddin, D.B. (2005b). Epidemiology of injuries in English professional rugby union: part 2 training injuries. British Journal of Sports Medicine, 39, 767-775.

Cohen, I. \& Stiff, M. (1979). Increased safety in rugby scrums. South African Medical Journal, 56, 525.

Garraway, W.M. \& Macleod, D. (1995). Epidemiology of rugby football injuries. Lancet, 345, 1485-1487.

Garraway, W.M., Lee, A.J., Hutton, S.J., Russell, E.B.A.W. \& Macleod, D.A.D. (2000). Impact of professionalism on injuries in rugby union. British Journal of Sports Medicine, 34, 348-351.

Gerrard, D.F. (1998). The use of padding in rugby union. Sports Medicine, 25, 329332.

Lee, A.J. \& Garraway, W.M. (1996). Epidemiological comparison of injuries in school and senior club rugby. British Journal of Sports Medicine, 30, 213-217. 
Marshall, S.W., Loomis, D.P., Waller, A.E., Chalmers, D.J., Bird, Y.N., Quarrie, K.L. \& Feehan, M. (2004). Evaluation of protective equipment for prevention of injuries in rugby union. International Journal of Epidemiology, 34, 113-118.

McIntosh, A. . (2005). Rugby Injuries. Medicine and Sport Science, 49, 120-139.

McIntosh, A. ., McCrory, P., Finch, C. . (2004). Performance enhanced headgear: a scientific approach to the development of headgear. British Journal of Sports Medicine, 38, 46-49

Milburn, P.D. (1990). The kinetics of rugby union scrimmaging. Journal of Sports Sciences, 8, 47-60.

Milburn, P.D. (1995). The rugby tackle: a time for review. Journal of Physical Education of New Zealand, 28, 9-15.

Milburn, P.D., Wilson, B.D. \& Chalmers, D.J. (2001). Evaluation of body protection clothing in rugby union. University of Otago.

Quarrie, K.L. \& Wilson, B.D. (2000). Force production in the rugby union scrum. Journal of Sports Sciences, 18, 237-246.

Quarrie, K.L., Cantu, R.C. \& Chalmers, D.L. (2002). Rugby union injuries to the cervical spine and spinal cord. Sports Medicine New Zealand, 32, 633-653.

Sumiya, T., Suzuki, Y., Kasahara, T. \& Ogata, H. (1998). Sensing stability and dynamic response of the F-Scan in-shoe sensing system: A technical note. Journal of Rehabilitation Research and Development, 35, 192-200.

Taylor, T.K.F. \& Coolican, M.R.J. (1987). Spinal chord injuries in rugby union. Medical Journal of Australia, 147, 112-118.

Wickiewicz, T.L., Edwards, J.C., Warren, R.F. \& Scarangella, S. (1993). Shoulder injuries in rugby union. In: Sports Medicine Congress, pp. 513-521. Edinburgh.

Wilson, B.D. (1998). Protective Headgear in Rugby Union. Sports Medicine, 25, 333337.

Wilson, B.D., Quarrie, K.L., Milburn, P.D. \& Chalmers, D.J. (1999). The nature and circumstance of tackle injuries in rugby union. Journal of Sports Science and Medicine in Sport, 2, 153-162. 